una catena d'eventi che hanno reso la resistenza agli antibiotici uno dei problemi sanitari di maggiore rilevanza mondiale. Inoltre, i ceppi MRSA sono divenuti una delle principali cause di infezione in ambiente ospedaliero. Questo studio ha come obiettivo la valutazione dell'antibiotico-resistenza di ceppi del genere Staphylococcus, appartenenti alle specie maggiormente isolate presso l'Ospedale di Latina e responsabili di infezioni invasive. La finalità di tale lavoro è comprendere meglio le basi epidemiologiche e biologiche del fenomeno.

Materiali e metodi Sono stati isolati, nel periodo che va dal 01/08/2002 al 31/07/2003, presso il Laboratorio Analisi dell'Ospedale "S. M. Goretti" di Latina, 311 stipiti di Staphylococcus spp.: 87 ceppi, pari al $27,97 \%$ del totale, rappresentati da stafilococchi coagulasi-positivi e 224 ceppi, pari al $72,03 \%$, identificati come stafilococchi coagulasinegativi. I ceppi sono stati isolati da materiali vari (tamponi faringei, tamponi urogenitali, t. auricolari, urine, sangue, espettorato) provenienti da pazienti ospedalizzati. Tutti gli stipiti sono stati identificati in base a proprietà biochimiche attraverso il sistema automatico Vitek (bioMérieux, Marcyl'Etoile, France) utilizzando le card GPI e testando la sensibilità agli antibiotici con card GPS 523 .

Risultati Gli stipiti maggiormente isolati sono rappresentati dallo Staphylococcus aureus con 87 ceppi $(27,97 \%)$ e dallo Staphylococcus epidermidis con 85 ceppi $(27.33 \%)$. Minor frequenza d'isolamento è stata riscontrata per Staphylococcus haemolyticus con 39 ceppi $(12,54 \%)$ e Staphylococcus hominis con 32 ceppi $(10,29 \%)$. Tutti gli stipiti isolati di Staphylococcus aureus sono risultati sensibili alla vancomicina e alla teicoplanina. La resistenza verso l'oxacillina (ceppi MRSA) è stata pari al $44,74 \%$, mentre la resistenza verso i macrolidi (eritromicina) è risultata del $55,26 \%$. La sensibilità verso i fluorochinoloni (ciprofloxacina) è risultata del $46,05 \%$, mentre il $96,05 \%$ dei ceppi sono risultati sensibili al Trimetoprim/Sulfametossazolo. Le percentuali di antibiotico-resistenza in Staphylococcus epidermidis sono spesso risultate diverse da quelle di Staphylococcus aureus. Degna di nota è la resistenza verso l'oxacillina, pari all' $85,71 \%$, il doppio di quella dello Staphylococcus aureus $(44,74 \%)$. Per quanto riguarda l'eritromicina la percentuale di ceppi resistenti è stata del $42,86 \%$, mentre il $19,05 \%$ dei ceppi ha presentato una sensibilità intermedia. La resistenza al trimetoprim/sulfametossazolo è stata del 71,43\%, mentre nello Staphylococcus aureus è risultata pari soltanto al $3,95 \%$. Inoltre, è da notare la maggiore resistenza verso la ciprofloxacina $(74,60 \%)$. Lo Staphylococcus haemolyticus ha mostrato una resistenza verso l'oxacillina pari all' $84,62 \%$; anche la resistenza verso la ciprofloxacina è stata elevata $(79,49 \%)$ mentre il trimeto$\mathrm{prim} / \mathrm{sulfametossazolo} \mathrm{è} \mathrm{risultato} \mathrm{sensibile} \mathrm{nel} 41,03 \%$ dei ceppi. L'eritromicina, infine, si è mostrata attiva soltanto verso il $17,85 \%$ dei ceppi testati. Gli stipiti di Staphylococcus hominis hanno mostrato una resistenza elevata sia verso la ciprofloxacina $(62,96 \%)$ che verso l'eritromicina $(81,48 \%)$ e l'oxacillina $(88,89 \%)$. Essi hanno presentato una elevata sensibilità verso la gentamicina $(81,48 \%)$ e la clindamicina $(96,30 \%)$.

Conclusioni Dal confronto di questi dati con quelli del territorio nazionale emerge l'estrema variabilità dell'antibioticoresistenza con conseguente necessità di personalizzare la terapia sulla base di dati certi riferiti all'agente patogeno e alle caratteristiche dell'ospite, riducendo l'impiego di antibiotici di ultima generazione solo in quei casi in cui non sono disponibili terapie di pari efficacia basate sull'utilizzo di molecole meno recenti e di più consolidato uso.

\section{P028 \\ ISOLAMENTO DI F. NECROPHORUM COME CAUSA DI SINDROME DI LEMIERRE}

Idda L.*, Castiglia N.*, Masala M.A. ${ }^{\circ}$, Burrai M. ${ }^{\circ}$, Milia S. ${ }^{\circ}$, Puggioni M. F. ${ }^{\circ}$, Merella G. ${ }^{\circ}$, Cherchi G.B.*

* Laboratorio di Analisi chimico cliniche e Microbiologia Ospedale Civile "SS. Annunziata" Sassari

- Divisione di Medicina I Ospedale Civile "SS. Annunziata" Sassari

La sdr. di Lemierre costituisce una infrequente ma grave complicanza di infezione faringo-tonsillare. Il caso da noi osservato riguarda un paziente di sesso maschile, dell'età di venti anni, forte fumatore e bevitore, dedito all'uso di droghe leggere; nel settembre del 2003 in seguito all'improvvisa insorgenza di faringodinia con febbre continuo-remittente con acme di $40.7^{\circ} \mathrm{C}$, il medico curante prescriveva terapia antibiotica con amoxicillina, sostituita in terza giornata con piperacillina/tazobactan. Il quadro clinico andava arricchendosi di nuovi sintomi quali dispnea con tosse scarsamente produttiva e adenite laterocervicale sinistra. Si decideva a questo punto il ricovero in ambito internistico dove si accertava la presenza di un esteso focolaio polmonare mediobasale destro, leucocitosi neutrofila, incremento della VES. Veniva a questo punto intrapresa terapia antibiotica: claritromicina per os, che veniva sostituita, per insuccesso, con ciprofloxacina. Emocolture seriate e tamponi faringei eseguiti presso il Laboratorio del nosocomio presso il quale era avvenuto il ricovero davano esiti negativi, mentre aumentava la estesa tumefazione latero-cervicale; si evidenziava mediante TC collo e torace una trombosi pressochè completa della giugulare interna sn., estendentesi alla vene succlavia e brachiocefalica omolaterale con infiltrati diffusi a livello polmonare. Il paziente ormai polipnoico, obnubilato, in stato soporoso, veniva trasferito all'Ospedale Civile SS. Annunziata di Sassari presso il reparto di Rianimazione. Venivano qui eseguite emocolture seriate e colture dell' aspirato bronchiale, con copertura antibiotica e.v. ad ampio spettro: teicoplanina, imipenem, aztreonam, fluconazolo ed eparina a basso peso molecolare. Dopo due giorni di trattamento, in seguito al miglioramento delle condizioni generali, il paziente veniva trasferito presso il reparto di Medicina dove veniva fatta diagnosi di sdr. di Lemierre ed impostata terapia e.v. con piperacillina-tazobactan, metronidazolo, gentamicina ed eparina a basso peso molecolare. A conferma del sospetto diagnostico dall'Unità Operativa di Microbiologia del Laboratorio Analisi veniva comunicata la positivizzazione di una delle emocolture inviate: la identificazione di un fusobacterium necrophorum, confermata poi anche con metodiche molecolari presso 1'Istituto Superiore di Sanità cui veniva inviato il ceppo, permetteva la definitiva conferma diagnostica, mentre il paziente andava incontro a rapido miglioramento delle condizioni generali.

P029

MIOCARDITE DA L. MONOCYTOGENES IN PAZIENTE CON OSTRUZIONE CORONARICA

\author{
Castiglia N.*, Idda L.*, Baule G. ${ }^{\circ}$, Melis A. ${ }^{\circ}$, Cherchi G.B.* \\ * Laboratorio di Analisi chimico cliniche e Microbiologia Ospedale \\ Civile "SS. Annunziata" Sassari \\ - Divisione di Medicina III Ospedale Civile "SS. Annunziata" Sassari
}

Il caso clinico oggetto del presente lavoro riguarda un 
paziente di sesso maschile, dell'età di 66 anni, con familiarità negativa per patologie cardiovascolari, ex fumatore, non storia di potus, né diabete e/o dislipidemie e/o patologie di rilievo all'anamnesi patologica remota. Ricoverato nel novembre 2003 presso la divisione di cardiologia dell'Ospedale SS. Annunziata di Sassari per sindrome coronaria acuta con ostruzione IVA prossimale, venivano riscontrati i seguenti valori ematochimici: VES 47, PCR 19, leucocitosi neutrofila ( $11.000 \mathrm{~GB}$ con $>70 \% \mathrm{~N})$; buona la funzionalità renale ed epatica. Ad un non riuscito tentativo di disostruzione coronarica, in attesa di intervento di by-pass aorto-coronarico si accompagnava rialzo termico con febbre elevata $>38^{\circ} \mathrm{C}$. All'ecocardiogramma, in assenza di focolai polmonari ed ecotomograzia addominale nella norma, si rilevava lieve ipertrofia parietale e presenza di versamento pericardico lieve-moderato; sulla base di tali riscontri si eseguivano numerosi prelievi venosi seriati per esami emocolturali e contemporaneamente veniva avviata terapia antibiotica ad ampio spettro con cefalosporine di terza generazione, teicoplanina per via ev ed acido acetilsalicilico ad alte dosi; a distanza di otto giorni non si rilevava alcun beneficio clinico ed anzi si manifestava progressivo aumento del versamento pericardico con segni di tamponamento cardiaco associato a grave anemia normocromica normocitica ( $\mathrm{Hb} 6.2$ g. x dl.). Trasferito presso il reparto di Medicina per ulteriori accertamenti e sottoposto ad emotrasfusione ed ulteriori esami colturali, si isolava da una delle emocolture inviate all' unità operativa di Microbiologia clinica del Laboratorio analisi ospedaliero una Listeria monocytogenes. Tale rilevazione rendeva possibile formulare la diagnosi di miocardite infettiva da Listeria e permetteva il corretto orientamento di una terapia antibiotica combinata mirata con penicillina ed aminoglicosidi per quattro settimane. Già in seconda giornata era possibile osservare una buona risposta clinica e, dopo un mese di terapia, il paziente veniva dimesso in ottime condizioni, con nessun segno di versamento pericardico; attualmente esita modesto ispessimento pericardico ed è stato programmato intervento di rivascolarizzazione miocardica.

\section{P030}

\section{TIPIZZAZIONE MOLECOLARE DI CEPPI DI MRSA MEDIANTE UTILIZZO DI RIBOPRINTER ${ }^{\circledR}$, RAPD E PFGE:ANALISI COMPARATIVA}

\section{Catalano A., Bonfitto M.G., Piana F., Cirillo D.M., Marchiaro G. \\ SC Microbiologia ASO San Giovanni Battista cso Bramante 88 - I0126 Torino}

Introduzione. Gli MRSA sono una causa persistente di infezioni nosocomiali a decorso grave. L'identificazione precoce di cloni "ospedalieri" è fondamentale per la sorveglianza negli ospedali di tutte le infezioni da MRSA. La caratterizzazione dei ceppi permette di rilevare tempestivamente eventuali microepidemie ed istituire le misure di controllo necessarie per arrestarle. In particolare la possibilità di ottenere un profilo genotipico (fingerprint) di ogni singolo isolato consente di delineare con certezza il percorso interumano dei ceppi. Negli ultimi anni sono state proposte diverse tecniche per la genotipizzazione tra le quali la ribotipizzazione automatica mediante Riboprinter ${ }^{\circledR}$, la Random Amplified Polymorphic DNA e la Pulsed Field Gel Electroforesis.

Scopo del lavoro. Lo scopo di questo lavoro è stata la valutazione delle 3 tecniche elencate sopra secondo diversi parametri quali la rapidità e la semplicità di esecuzione, la capacità discriminatoria e la riproducibilità dei dati.
Materiali e metodi. A tale scopo sono stati tipizzati 77 ceppi di MRSA isolati da materiali patologici, 69 dei quali provenienti da reparti diversi dell'Ospedale Maggiore San Giovanni Battista di Torino e 8 da ospedali esterni.

La tipizzazione in PFGE è stata eseguita mediante l'utilizzo dell'enzima Sma1 e seguendo il protocollo descritto altrove, mentre il sistema Riboprinter ${ }^{\circledR}$ l'enzima EcoR1.

La RAPD è stata effettuata con i primer forniti dal kit ReadyTo-Go $^{\mathrm{TM}}$ RAPD Analysis Kit (Amersham Biosciences) e seguendo le procedure indicate dal produttore.

L'analisi dei patterns è stata effettuata mediante il software Bionumerics 2.5 per la rivelazione dei clusters.

Risultati. L'analisi eseguita mediante RAPD ha permesso di individuare 35 profili differenti (costituiti da un numero di bande compreso tra 1 e 5), all'interno dei quali 56 ceppi sono stati inclusi in 14 clusters con indice di silmilarità del $100 \%$ cioè in raggruppamenti costituiti da profili identici. I 14 clusters sono costituiti 1 da 11 ceppi; 1 da 9; 1 da 7; 1 da 5; 1 da $4 ; 2$ da 3 e 7 da 2 ceppi. L'indice di clusterizzazione è risultato essere $72.7 \%$ (56 ceppi/77)

L'analisi mediante PFGE ha evidenziato, invece, 45 pulsotipi differenti all'interno dei quali 45 ceppi sono stati inclusi in 14 clusters (IS $=100 \%$ ) formati a loro volta da 1 clusters da 7 ; 1 da $6 ; 1$ da $4 ; 6$ da 3 e 5 da 2. L'indice di clusterizzazione della PFGE è $58.4 \%$

La ribotipizzazione automatica mediante Riboprinter ${ }^{\circledR}$ ha individuato 16 ribotipi. I cluster rilevati dal Riboprinter ${ }^{\circledR}$ sono stati 7: il ribotipo 19-S-6 comprende 16 ceppi, il 90-S1 37, il 207-S-2 3, 4 il 90-S-8, 2 il 210-S-4, 4 il 105-S-7, 2 il 19-S-1. L'indice di clusterizzazione è stato del $88.3 \%$.

Esperimenti ripetuti hanno evidenziato l'ottima riproducibilità della PFGE e del Riboprinter ${ }^{\circledR}$ mentre la riproducibilità ottenuta mediante la tecnica del random priming si è rivelata insufficiente. Per quanto riguarda i tempi di esecuzione sono risultati prolungati per la PFGE (una settimana) mentre la metodica RAPD permette di ottenere una tipizzazione in una giornata lavorativa; infine la tipizzazione mediante Riboprinter® abbina ai tempi rapidi di esecuzione la quasi completa automatizzazione.

Per quanto riguarda i costi essi risultano modesti per la RAPD, medio-alti per la PFGE, mentre sono rilevanti per il sistema automatizzato Riboprinter ${ }^{\circledR}$

Conclusioni. Lo studio comparato di tali metodiche ci ha permesso di confermare quanto noto in letteratura: la metodica PFGE è il "gold standard" per lo studio epidemiologico degli MRSA, mentre il Riboprinter ${ }^{\circledR}$, pur essendo di rapida e semplice esecuzione ed evidenziando un'ottima riproducibilità del sistema, si è dimostrata una tecnica meno discriminante $\mathrm{i}$ cui risultati devono essere confermati dalla PFGE. Il sistema automatico può essere utilizzato come metodica di screening su numerosi ceppi. Infine la RAPD si è dimostrata una metodica applicabile a epidemie di differenti patogeni.

\section{BIBLIOGRAFIA:}

1. Tenover F.C., Arbeit R.D., Goering R. V., Mickelsen P.A, Murray B.E, Persing D.H., Swaminathan B. Interpreting chromosomal DNA restriction patterns produced by pulsed-field gel electrophoresis; criteria for bacterial strain typing. J Clinic Microbiology. 1995; 33:22332239.

2. Prevost G., Jaulhac B., Piemont Y. DNA fingerprint by pulsed-field gel electrophoresis is more effective then rybotyping in distinguishing among methicillin-resistant S.aureus isolates J Clin Microbiology 1992; 30: 967-73.

3. Saulnier P., Bourneix C., Prevost G., Andremont A. Random amplified polymorphic DNA assay is less discriminant than pulsed-field gel electrophoresis for typing strain of methicillin-resistant S.aureus J Clin Microbiology. 1993; 31: 1964-70. 